\title{
Therapiebegrenzung, Therapieverzicht und Therapieabbruch - das BGH-Urteil vom 25.06.2010
}

\author{
Gerd Richter
}

Online publiziert: 13 . Oktober 2010

(C) Springer-Verlag 2010

Mit dem Grundsatzurteil des BGH vom 25. Juni 2010 (2 StR 454/09) ist die Situation hinsichtlich der Sterbehilfe durch Unterlassen, Begrenzen oder Beenden einer bereits begonnenen medizinischen Behandlung bei rechtfertigendem tatsächlichen oder mutmaßlichen Patientenwillen im Anschluss an die Novellierung des Betreuungsrechts (drittes Gesetz zur Änderung des Betreuungsrechts vom 29. Juli 2009) nochmals rechtlich geklärt worden; insbesondere unter Aufhebung der rechtsnormativen Differenz von Unterlassen und aktivem Tun.

Diesem Grundsatzurteil lag die Verurteilung eines Rechtsanwalts/Medizinrechtlers zugrunde, die das Landgericht Fulda ausgesprochen hatte. In der Begründung des Landgerichts sollte es sich im konkreten Fall um den versuchten Totschlag durch aktives Tun gehandelt haben. Im konkreten Fall ging es, entsprechend dem BGH-Urteil, um eine 76jährige Patientin, die seit Oktober 2002 nach einer Hirnblutung im Wachkoma lag und in einem Pflegeheim versorgt wurde, u. a. mittels enteraler Ernährung über eine PEG. Entsprechend einer mündlichen Äußerung im September 2002 hatte die Patientin ihren Kindern i. S. eines Behandlungswunsches mitgeteilt, dass sie im Falle einer Bewusstlosigkeit keine lebensverlängernden Maßnahmen in Form von künstlicher Ernährung und Beatmung für sich in Anspruch nehmen wolle. Auf diesem Hintergrund bemühten sich die Kinder, die im Intervall als Betreuer eingesetzt worden waren, mit Hilfe eines Rechtsanwalts und mit Unterstützung des betreuenden Hausarztes um die Einstellung der künstlichen, enteralen Sondenernährung. Nach Kompromissbildung mit der Heimleitung beendeten die Kinder als Betreuer die Sondenernährung und reduzierten die Flüssigkeitszufuhr. Allerdings wies die Geschäftsleitung die Heimleitung am nächsten Tag an, die künstliche Ernährung umgehend wieder aufzunehmen. In dieser Situation gab der Rechtsanwalt telefonisch den Kindern den Rat, die PEG-Sonde direkt an der Austrittsstelle abzuschneiden. Dieses Geschehen wurde unmittelbar nach der Handlung entdeckt. Nach Einschaltung der Polizei durch die Heim-

G. Richter $(\triangle)$

Fachbereich Medizin, Dekanat/Ethikkommission,

Philipps-Universität Marburg, Baldinger Strasse,

35032 Marburg, Deutschland

E-Mail: richterg@staff.uni-marburg.de 
leitung wurde die Patientin auf Anordnung eines Staatsanwaltes in eine Klinik eingewiesen, in der eine PEG-Neuanlage durchgeführt wurde. Die Patientin verstarb 14 Tage später aufgrund ihrer Grunderkrankungen. Obwohl das Landgericht Fulda die Anordnung der Wiederaufnahme der künstlichen Ernährung durch die Heimleitung als rechtswidrigen Angriff gegen die körperliche Integrität und das Selbstbestimmungsrecht der Patientin angesehen hat, begründete das Gericht die Verurteilung des Rechtsanwaltes damit, dass die durchgeführte Handlung (identisch mit dem Rat zur Handlung durch den Rechtsanwalt) nicht Unterlassen, sondern ein aktives Tun sei. Nach Auffassung des Landgerichts haben die Voraussetzungen einer zulässigen ,passiven“ Sterbehilfe nicht vorgelegen, da diese nach Gerichtsauffassung ausschließlich im Unterlassen zu sehen sind. Die Aufhebung des Landgerichtsurteils und der Freispruch des Angeklagten durch den Bundesgerichtshof werden im Urteil vom 25. Juni 2010 damit begründet, dass die Grenze zwischen erlaubter Sterbehilfe und einer strafbaren Tötung nicht sinnvoll nach Maßgabe einer naturalistischen Unterscheidung von aktivem und passivem Handeln bestimmt werden kann. In dieser Hinsicht macht der Bundesgerichtshof in seiner Entscheidung darauf aufmerksam, dass der zugrunde liegende Lebenssachverhalt von entscheidender Bedeutung ist. Dies stimmt mit der ethischen Analyse von D. Birnbacher überein, der bereits 1995 festgestellt hat, dass ,,nicht der Handlungs- oder Unterlassungscharakter des zu beurteilenden Verhaltens selbst den Ausschlag gibt, sondern die moralisch relevanten Unterschiede in den externen Faktoren, von denen angenommen wird, dass sie mit der Handeln-Unterlassen-Unterscheidung kovariieren“" ([1], S. 128).

Sterbehilfe durch Unterlassen, Begrenzen oder Beenden einer begonnenen medizinischen Behandlung ist gerechtfertigt durch den tatsächlichen oder mutmaßlichen Patientenwillen, so dass es dadurch dem Patienten ermöglicht wird, an seiner ihm zugrunde liegenden Erkrankung zu versterben. Dies ist weiterhin rechtlich klar abgegrenzt von einer vorsätzlich lebensbeendenden Handlung, bei der der Patient nicht an der ihm zugrunde liegenden Erkrankung verstirbt, sondern an jenem gezielten Eingriff, der einer Tötung auf Verlangen entspricht, welche auch nie durch Verlangen und Einwilligung rechtfertigbar ist (§216 StGB). In diesem Zusammenhang sollte auch die Terminologie dieser Sachverhalte neu überdacht und Abstand von den Begrifflichkeiten einer ,passiven“ oder ,,aktiven“ Sterbehilfe genommen werden.

Zusammenfassend ist mit diesem BGH-Urteil vom 25. Juni 2010 auf dem Hintergrund der Novellierung des Betreuungsrechts durch das Gesetz vom 29. Juli 2009 („Patientenverfügungsgesetz") eine Rechtssicherheit geschaffen worden, die zum einen nicht entscheidungsfähigen Patienten mit Hilfe eines Betreuers/Bevollmächtigten zur Durchsetzung ihres Selbstbestimmungsrechts verhelfen soll und zum anderen eine Verfahrenssicherheit für die behandelnden Ärzte ermöglicht in der Feststellung des Patientenwillens und der sich daraus ableitenden Umsetzung indizierter medizinischer Maßnahmen entsprechend dem vorausverfügten oder mutmaßlichen Patientenwillen. Rechts- und Verfahrenssicherheit für den Arzt im Rahmen dieser gesetzlichen Vorgaben bei der Sterbehilfe ist allerdings nur mittels biographischer Arbeit an der Patientengeschichte und dem gemeinsamen Gespräch mit dem Betreuer oder dem Bevollmächtigten zu erreichen, so dass dieser Sachverhalt die Relevanz des ärztlichen Gesprächs nochmals deutlich werden lässt.

\section{Literatur}

1. Birnbacher D (1995) Tun und Unterlassen. Reclam, Stuttgart 